\title{
GLOBAL STABILITY CONTROL OF POWER SYSTEMS
}

\author{
Joseph S. K. Leung and David J. Hill \\ Department of Electronic Engineering, \\ City University of Hong Kong \\ (e-mail: joeleung, eedavid@cityu.edu.hk).
}

\begin{abstract}
This paper introduces an adaptive coordination scheme to optimally enhance the transient stability, voltage security and frequency damping of a power system network by scheduling the parameters of the available controllers. A set of real-time stability indicators are used to reflect the power system stabilities. The relative significances of a group of controllers to the power system are used as a reference to schedule new controller parameters. This scheme effectively allocates power system resources to handle different stability issues in different time frames. The results suggest that power system security can be further enhanced by means of coordinated control. Copyright (C) 2005 IFAC
\end{abstract}

Keywords: Adaptive control, Small signal stability, Transient stability, Voltage stability.

\section{INTRODUCTION}

Power systems are becoming more complex. Traditional vertically integrated power systems are being replaced by market-based operation after deregulation. Furthermore, the introduction of independent power producers (IPP) and powerelectronic loads have also complicated the operation of the grid. These introduce new uncertainties and nonlinearities and so it becomes more difficult to predict the behaviour of power systems. The equilibrium point is not only determined by the existing physical constraints, such as generator supply, load demand and transmission line thermal limits, but also by economic considerations. While the operation and the interconnection of power systems are evolving, few changes appear in the mainstream control of power system, i.e. most controllers are still designed for handling predictable local problems only. They rarely coordinate together to resolve any particular network problem, not to mention problems of an unusual nature.

The research is sponsored by Hong Kong Research Grant Council under CityU 1297/03E.
Power system stability issues are not mutually exclusive. Major system disturbances give rise to a combination of transient instability, voltage instability and/or frequency oscillation. Other dynamic problems may arise, but this paper focuses on these issues for illustration of a general technique. Careful examination of the instability "symptoms" is needed before taking any remedial actions. On the other hand, the selection of remedies also requires attention. While one controller action is selected to be beneficial to one kind of stability problem, it may deteriorate the power system in other respects. There have been qualitative studies (Kundur, 1994; Kundur et al., 2004) showing that some controller interactions could degrade the power system stability at some circumstances in which they are not designed for.

This paper studies a scheme for coordinated security control. The concept of global control (Hill, et al., 2001) evolved from previous works by the authors (Hill, 2001; Guo, 2001; Leung, 2002; Leung, 2004). In (Hill, et al., 2001), a novel global control strategy was introduced for coordinated transient stability and voltage regulation by excitation. In (Guo, et al., 2001), a framework was developed for more general 
schemes involving coordination of many controls with diverse goals. In (Leung, 2002, 2004), the framework was refined, applied to a benchmark test system and covered more different network scenarios. In this paper, the methodology is further extended. The "relative effectiveness" of three controllers, namely an excitation controller, power system stabilizer (PSS), and static VAr compensator (SVC) are firstly evaluated for the capabilities to resolve transient instability, voltage instability and small signal oscillatory problems. A linearized "sensitivity matrix" is formed which is used by the optimizer later as a reference for control parameter scheduling. Power system signals are sensed and processed for stability cost calculations. The severities of the stability issues are compared based on their weighted costs and a new set of controller parameters are optimally calculated with the aim of reducing the total stability cost. The simulation results for a benchmark system show that this global control scheme is effective and robust and provides significant stability improvement for all kinds of system disturbances.

The paper is structured as follows. The test system is described Section 2. The formulation of the global control system and its various indicators are presented in Section 3. Simulation and results for different scenarios are provided in Section 4, followed by conclusions in Section 5 .

\section{SYSTEM DESCRIPTION}

Fig. 1 shows the diagram of a modified version of the two-area system described in (Klein, et al., 1991). The modifications are as follows:

- Line 6-7 and line 9-10 are split into double circuits

- Line 7-8 and line 8-9 are turned into 4-line circuits

- Two SVC's are installed at Bus 7 and Bus 9 The $0.5-0.6 \mathrm{~Hz}$ inter-area mode of oscillation, which has the lowest damping factor, is chosen as the target for damping in this paper (Kundur, 1994; Klein, 1991). All the dynamic data of the generators and static data of the system remain unchanged from that given in (Klein, et al., 1991).

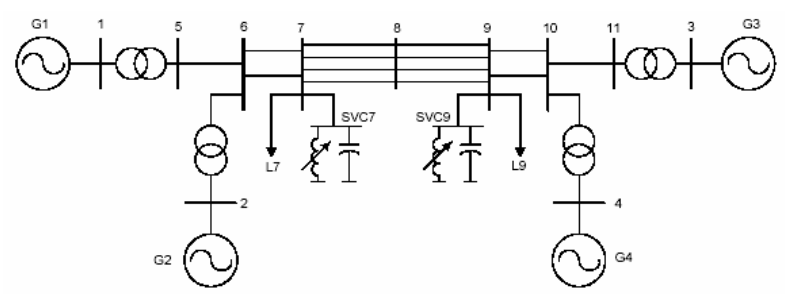

Fig. 1. A modified 2-Area System

Throughout this paper, a number of contingencies are referred to. The descriptions of them are as follows:

C1: 3-Phase fault at Bus 7. Fault is cleared by tripping line $7-8$ at 0.1 second later;

C2: 3-Phase fault at Bus 8 . Fault is cleared by tripping line 7-8 and line 8-9 at 0.1 second later;

C3: 3-Phase fault at Bus 9. Fault is cleared by tripping line 8-9 at 0.1 second later;

\section{C4: $\quad 10 \%$ load increase at Bus 9.}

\subsection{General Idea}

\section{GLOBAL CONTROL}

The goal of the adaptive coordinated control proposed is to maintain system stability under all operating conditions, especially unforeseeable ones. It is a framework within which a variety of methods and different controllers can be coordinated to handle complex system problems to the extent allowed by the available information communication technology (ICT). It offers the possibility to coordinate control actions across the whole system geographically and for all operating situations in an adaptive way (Hill, 2001; Guo, 2001).

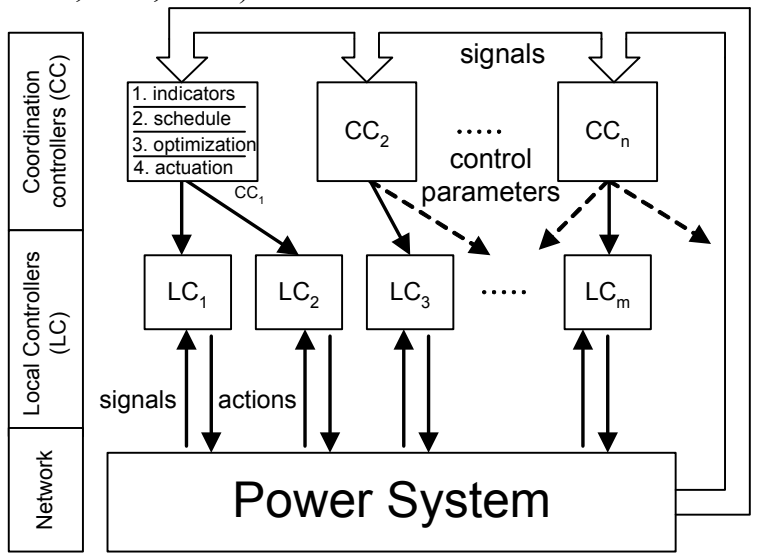

Fig. 2. Adaptive coordination control framework

A diagram of this control framework is shown in Fig. 2. This system-wide control has four main tasks, namely: 1) indicator processing, 2) scheduling, 3) optimization and 4) actuation. The control is represented with decentralized structure to emphasize it is typically not centralized (due to ICT limitations). The signal processing layer collects and processes network information and transforms it into meaningful signals such as stability indicators. The coordination controller will then determine the severity of each kind of instability, i.e. to decide whether the system is in the proximity of transient, voltage and/or frequency instability. Accordingly, a group of control actions (typically of the switching type) which have the capacity, will be scheduled with further tuning, to overcome the instability. After the optimization process, which allows tuning of control parameters, a set of weighted parameters will be transmitted to the corresponding local controllers for actuation.

\subsection{Controller Sensitivity Tests}

Power system controllers are mostly designed to satisfy some kind of primary objectives. For example, PSS is designed for damping and SVC is for voltage support. However, their contributions to other types of stability controls are also valuable. Information like the relative strength of each controller is required for the global control system to formulate suitable switching actions.

Transient Stability Assessment The transient stability index of a multi-machine system can be simply 
computed by using the extended equal area criteria (EEAC) (Xue, et al., 1989). This method separates the system generators into two groups, i.e. the critical cluster and the rest. The cluster of critical machines is identified as the group of "candidate" critical machines which give the smallest critical clearing time (CCT). By performing a critical cluster centre of inertia (CCCOI) transformation, the system is mapped into a fictitious SMIB system. The stability index is obtained by calculating the difference between the "acceleration area" and "deceleration area" in the corresponding EEAC assessment. The index is defined as:

$\eta_{t s}=\left\{\begin{array}{l}\frac{A_{d e c}-A_{\text {inc }}}{A_{\text {dec }}} \cdot 100 \% \\ \text { if the system is "stable" }\left(A_{\text {dec }}>A_{\text {inc }}\right) \\ \frac{A_{\text {dec }}-A_{\text {inc }}}{A_{\text {inc }}} \cdot 100 \% \\ \text { if the system is "unstable" }\left(A_{\text {dec }}<A_{\text {inc }}\right)\end{array}\right.$

where

$A_{\text {inc }}=$ kinetic energy increasing area (during fault)

$A_{d e c}=$ kinetic energy decreasing area (post fault)

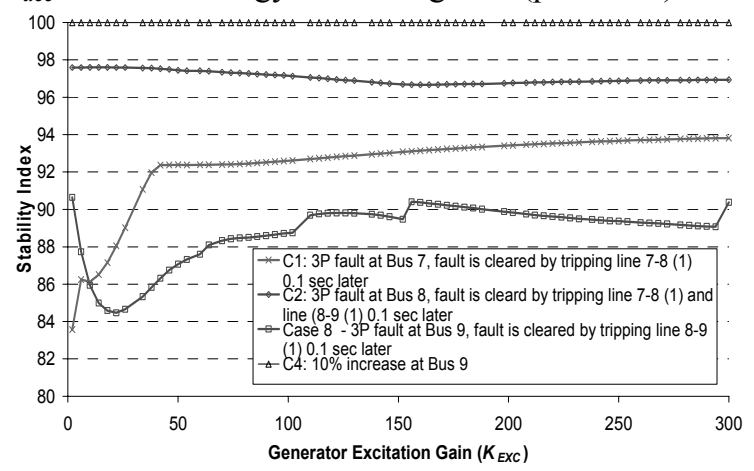

Fig. 3a. Transient stability index vs. excitation gains

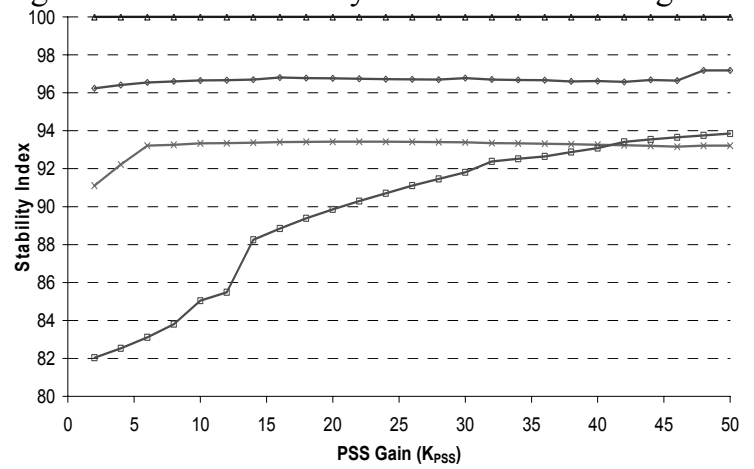

Fig. 3b. Transient stability index vs. PSS gains

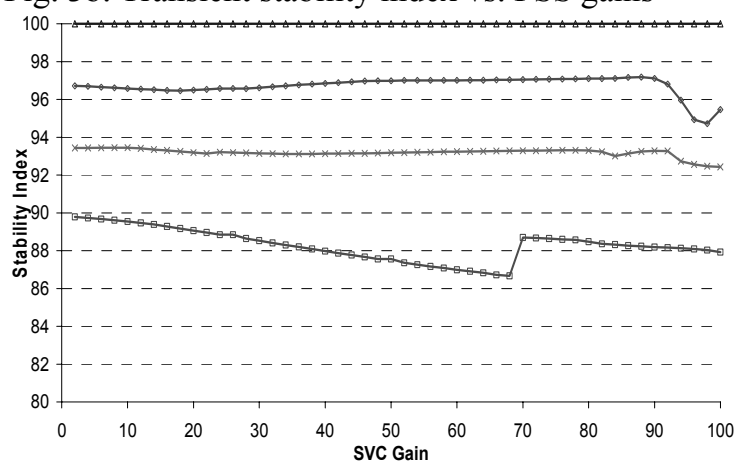

Fig. 3c. Transient stability index vs. SVC gains

The transient stability assessments for the excitation system, PSS and SVC are performed by using the
Powertech's Transient Stability Assessment Tool (TSAT). The initial gain values of the exciter, PSS and SVC are set to be 200, 30 and 200 respectively. By varying the gain of one kind of controller at a time, the transient stability indices are found. The results are shown in Fig. 3a-3c. It is noted that:

- There are discontinuities in the stability indices. This is because the EEAC algorithm separates different machines into different clusters according to the degrees they are affected. Different combinations of critical generators will therefore change the inertia base of the calculation;

- Fig. 3a shows that increased gain in the excitation system does not always increase the stability index; and Fig. $3 \mathrm{~b}$ shows that increase in PSS gain does not necessarily worsen the transient stability. It depends on the particular situation. However, these two figures show that the actions of the excitation system and PSS in transient stability tend to be oppositional;

- Increase in SVC gain does not improve the transient stability - see Fig. 3c.

Transient Voltage Stability Assessment Voltage stability problems are generally caused by deficiency of reactive power in some sense. By estimating the "distance to the proximity of voltage collapse", many stability indices have been proposed (Canizares, et al., 2002). In this paper, voltage security (or transient voltage stability) which describes the deviation of transmission system voltage from its nominal value is studied (Hiskens and Hill, 1989). Other forms of voltage problem can also be included.

Based on signal energy analysis (De Tuglie, et al., 2000), a transient voltage instability index, which measures the normalized distance of the transient voltage from the steady-state value, is chosen. The equation is stated below:

$$
\eta_{t v s}=\frac{\sum_{i=n_{c l}}^{n_{T}} \sum_{j=1}^{k}\left(\frac{V_{i, j}-\overline{V_{j}}}{V_{i, j}}\right)^{2}}{\left(n_{T}-n_{c l}\right) k}
$$

where

$\eta_{t v s}$ is the transient voltage instability index;

$n_{c l}$ is the time step at the clearing time;

and the subscript $j$ denotes buses and $i$ denotes the time step.

Inspection on Fig. 4a, $4 \mathrm{~b}$ and $4 \mathrm{c}$ reveals that:

- The transient voltage instability index increases monotonically in a close-to-linear fashion as the excitation gains increase from 100 to 300 - see Fig. 4a;

- $\quad$ Fig. 4b shows that change in PSS gains has less effect on transient voltage stability;

- The SVC is the most effective device for transient voltage stability improvement see Fig. 4c;

It is therefore concluded that a reduction in excitation gain and an increase in SVC gain can improve transient voltage stability. 


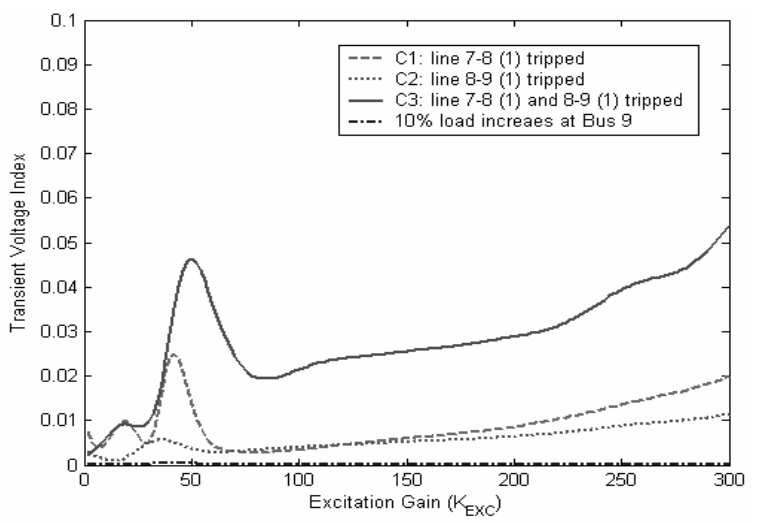

Fig. 4a. Transient voltage index vs. excitation gains

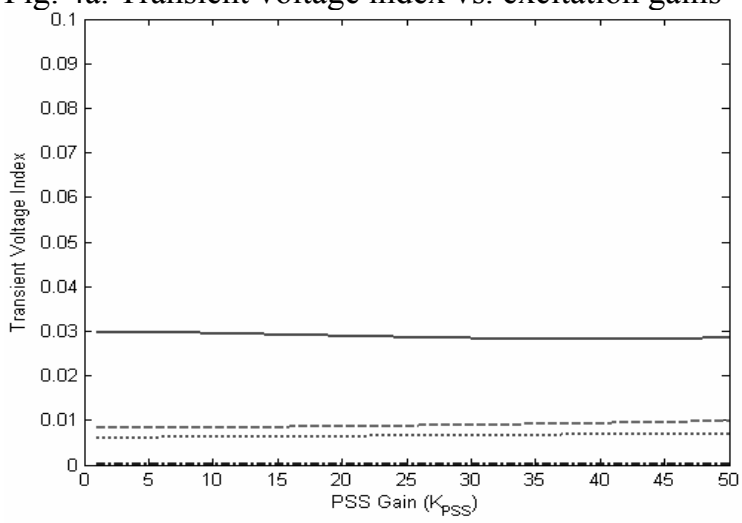

Fig. 4b. Transient voltage index vs. PSS gains

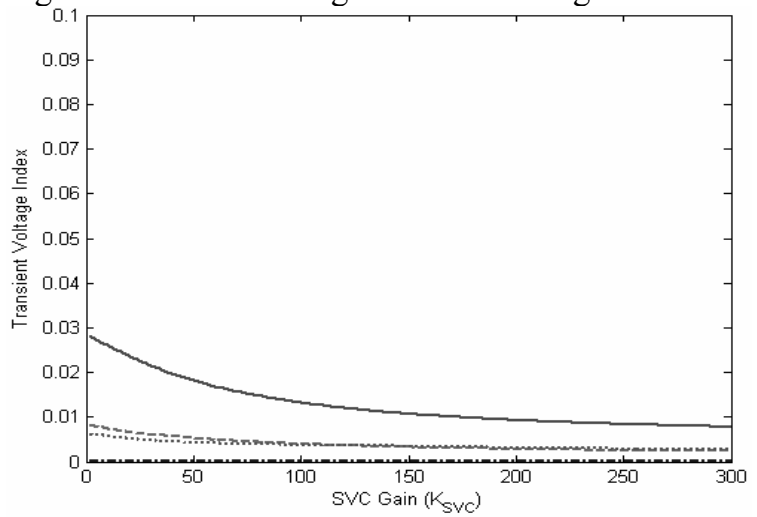

Fig. 4c. Transient voltage index vs. SVC gains

Small Signal Stability Assessment Different modes of oscillation are present in a multi-machine power system. Each mode is characterized by its natural frequency and damping factor. Prony's method (Lawrence Marple, 1987) is a common way to extract the frequency information in real time. It considers the sampled data as a linear combination of exponentials:

$$
\hat{x}[n]=\sum_{k=1}^{p} A_{k} e^{\left[\left(\alpha_{k}+j 2 \pi f_{k}\right)(n-1) T+j \theta_{k}\right]}
$$

for $1 \leq n \leq N$, where

$T$ is the sample interval,

$A_{k}$ is the amplitude,

$\alpha_{k}$ is the damping factor,

$f_{k}$ is the sinusoidal frequency,

$\theta_{k}$ is the sinusoidal initial phase.

The $p$-exponent discrete-time function of (3) can be expressed in the form:

$$
\hat{x}[n]=\sum_{k=1}^{p} h_{k} z_{k}^{n-1}
$$

The damping factor $(\alpha)$ corresponding frequency $(f)$ and damping index $\left(\eta_{s s}\right)$ can be calculated by:

$$
\begin{aligned}
& \alpha_{i}=\frac{\ln \left|z_{i}\right|}{T} \sec ^{-1} \\
& f_{i}=\tan ^{-1} \frac{\operatorname{Im}\left\langle z_{i}\right\rangle / \operatorname{Re}\left\langle z_{i}\right\rangle}{2 \pi T} H z \\
& \eta_{s s}=\frac{\alpha}{100} \%
\end{aligned}
$$

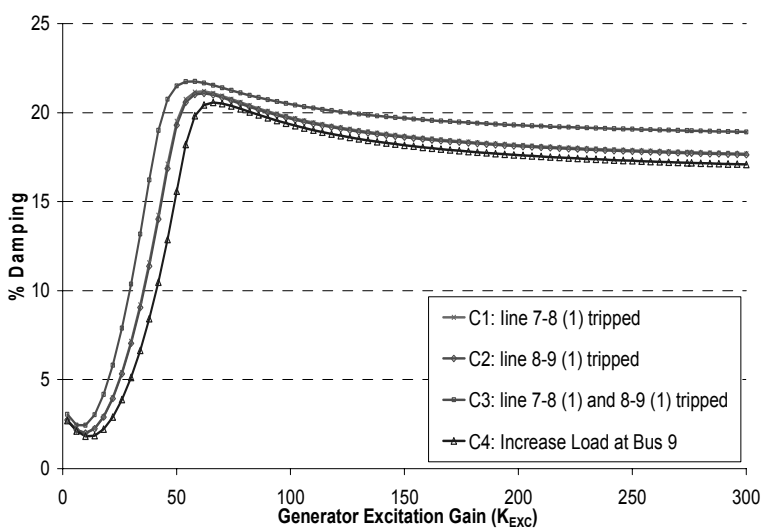

Fig. 5a. Small signal stability index vs. excitation gains

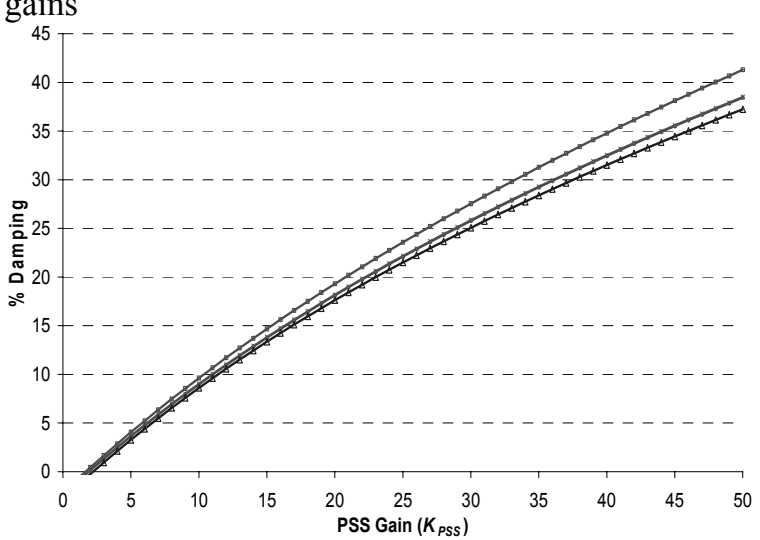

Fig. 5b. Small signal stability index vs. PSS gains

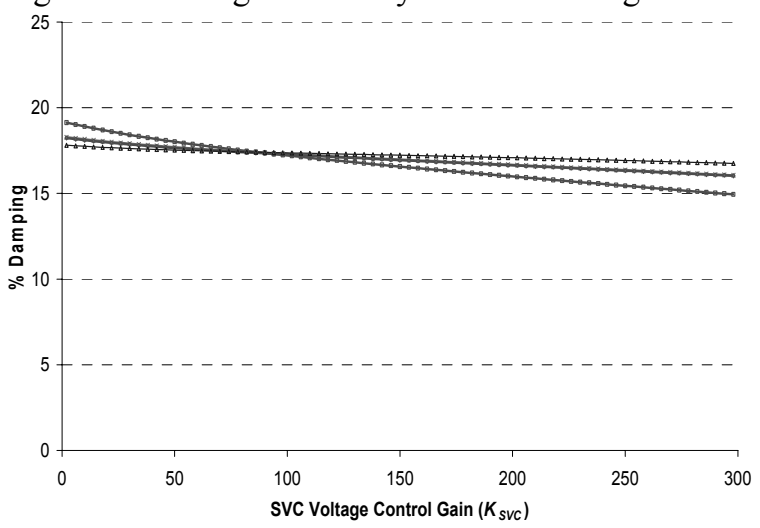

Fig. 5c. Small signal stability index vs. SVC gains

Fig. $5 \mathrm{a}, 5 \mathrm{~b}$ and $5 \mathrm{c}$ show the $\%$ damping variations with different controller gains. It is noted that the damping ratio of the inter-area mode increases almost linearly with the increase of PSS gains. On the other hand, while the damping factor also increases when the excitation gain increases from 0 to about 60 , it begins to decrease monotonically after the peak. Increase of SVC gains also decreases the damping ratio in this case. 


\subsection{Global Control Switching}

The global control switching law has the following parts:

- Controller sensitivity matrix

- Real-time stability indicators

- Optimization Function

Sensitivity Matrix The capabilities of the power system controllers for different stabilities can be summarized in the "sensitivities matrix" measured at a nominal operating point. The general form is:

$$
\Delta C=A \cdot \Delta K
$$

where $C$ is the cost, $A$ is the gradient between cost and controller gain $K$. (Note that $K$ in general can be any controller parameter, such as for load shed.) In this paper, the following $3 \times 3$ matrix is used:

$$
\left[\begin{array}{c}
\Delta C_{t s} \\
\Delta C_{t s s} \\
\Delta C_{s s}
\end{array}\right]=\left[\begin{array}{lll}
a_{11} & a_{12} & a_{13} \\
a_{12} & a_{22} & a_{23} \\
a_{31} & a_{32} & a_{33}
\end{array}\right]\left[\begin{array}{l}
\Delta K_{E X C} \\
\Delta K_{S V C} \\
\Delta K_{P S S}
\end{array}\right]
$$

Stability Costs While the transient stability index from EEAC is a well-accepted indicator for transient stability, it cannot provide a continuous assessment of the overall status of the generator angles. Based on the CCCOI procedure, a modified method to measure the overall rotor angle deviation is thus proposed:

$$
\Delta \delta_{s a}=\delta_{s}-\delta_{a}=\frac{\sum_{i \in S} M_{i} \delta_{i}}{\sum_{i \in S} M_{i}}-\frac{\sum_{j \in A} M_{j} \delta_{j}}{\sum_{j \in A} M_{j}}
$$

where

$M_{i}$ and $M_{j}$ are the moments of inertia of the $i$-th and $j$-th machine in the $S$-cluster and $A$-cluster respectively; $\delta_{i}$ and $\delta_{j}$ are the rotor angles of the $i$-th and $j$-th machine in the $S$-cluster and $A$-cluster respectively.

To represent the angle excursion as a cost to the power system, the following function is used:

$$
C_{t s}=\frac{\Delta \delta_{s a}}{\delta_{c}}
$$

where $\delta_{c}$ is the critical angle of the system determined by the EEAC (Xue, et al., 1989).

The value of $C_{t s}$ is between 0 and 1 . The transient stability "limit" is defined by $C_{t s}=1$.

Since the transient voltage instability index measures how "voltage unstable" the system is, it can be directly used as the cost to be minimized for transient voltage stability, i.e.

$$
C_{t s s}=\min \left(\eta_{v s s}, \lambda\right)
$$

Note that the value of the transient voltage stability cost is kept below $\lambda$. During phase-to-earth faults, voltage would momentarily drop to zero. The voltage deviation (thus voltage stability cost) from the nominal value would be large when compared with other stability costs. However, transient stability cost is considered to be the most important during the fault period. For illustration here $\lambda$ is taken as 0.3 which covered the voltage fluctuations encountered. The small signal stability cost is the normalized deviation of the damping factor of the frequency from the targeted one:

$$
C_{s s}=\left|1-\frac{\eta_{s s}}{\eta_{s s 0}}\right|
$$

where $\eta_{s s 0}$ is the target damping factor.

The maximum value of $C_{s s}$ is 1 and the minimum value is limited to 0 , even if $\eta_{s s} \geq \eta_{s s 0}$.

Optimization The determination of new controller parameters is formulated as a constrained optimization program. A quadratic objective function is proposed:

$$
\min J=\left(\alpha_{t s} C_{t s}{ }^{2}+\alpha_{t s s} C_{t v s}{ }^{2}+\alpha_{s s} C_{s s}{ }^{2}\right)
$$

and

$$
\alpha_{\mathrm{ts}}+\alpha_{\mathrm{ss}}+\alpha_{\mathrm{tvs}}=1
$$

Coefficients $\alpha_{t s}, \alpha_{s s}$ and $\alpha_{t v s}$ are the weights for the corresponding stability indices. They are determined with the aim to balance the contribution of each kind of stability in different time intervals. The costs $C_{t s}$, $C_{t v s}$ and $C_{s s}$ are estimated in real-time and are dependent on the existing power system state variable $x_{0}$, algebraic variables $y_{0}$, disturbances $u_{0}$, controller parameters $\mu_{0}$, and trial parameter increment $\mu^{+}$. Thus the estimated costs can be written:

$$
\begin{aligned}
& \hat{C}_{t s}=C_{t s}\left(x_{0}, y_{0}, u_{0}, \mu_{0}\right)+\Delta C_{t s}\left(\mu^{+}\right) \\
& \hat{C}_{t v s}=C_{t v s}\left(x_{0}, y_{0}, u_{0}, \mu_{0}\right)+\Delta C_{t v s}\left(\mu^{+}\right) \\
& \hat{C}_{s s}=C_{s s}\left(x_{0}, y_{0}, u_{0}, \mu_{0}\right)+\Delta C_{s s}\left(\mu^{+}\right)
\end{aligned}
$$

and

$$
\mu=\mu_{0}+\mu^{+}
$$

Thus, in terms of $\mu, J$ can be expressed as:

$$
J=J\left(x_{0}, y_{0}, u_{0}, \mu\right)
$$

This objective function is subject to two constraints:

(a) Upper and lower bounds:

$$
\mu_{l b} \leq \mu \leq \mu_{u b}
$$

(b) Limited rate of change in controller gains:

$$
\frac{d \mu}{d t} \leq r
$$

The inequalities can be converted into the following general form:

$$
G_{i}(\Delta \mu) \leq 0
$$

\section{SIMULATION AND RESULTS}

In this simulation, the load at Bus 9 is increased by $10 \%$ at $\mathrm{t}=2.0 \mathrm{~s}$. Then at $\mathrm{t}=6.0 \mathrm{~s}$, a fault occurs at Bus 8 . The fault is cleared by tripping one line $8-9$. This portrays a scenario where multiple events occur. The effects of the global coordinated control scheme are demonstrated and compared with a conventional method where no global control is used, i.e. parameters are fixed at values determined in (Kundur, 1994). The results are shown in Fig. 6a-d. Fig. 6a shows that the angle deviation between machine 1 and 3 settles faster under global control. Damping control only swamps in at the end. The cost comparisons in Fig. $6 \mathrm{~b}$ and $6 \mathrm{c}$ also confirm that transient stability, transient voltage stability and damping are improved by the control scheme. The controller gains in Fig. 6d highlight the point that the controllers can automatically reschedule their parameters for successive events. 


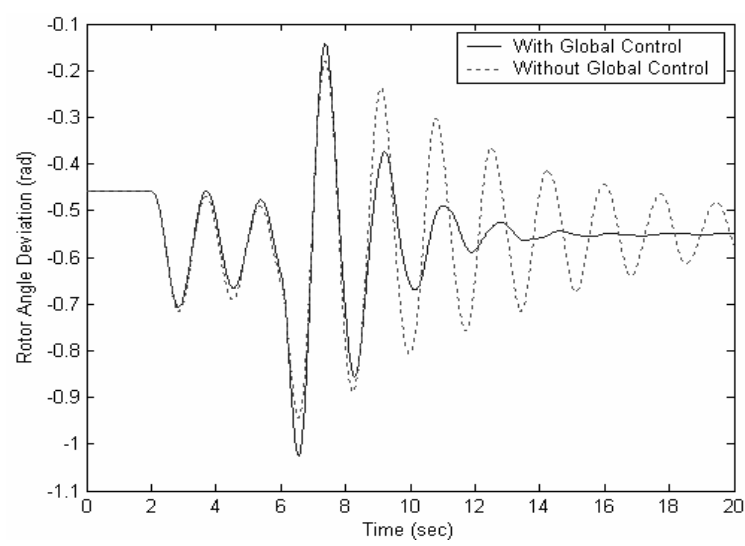

Fig. 6a. Rotor angle deviation among machines $1 \& 3$

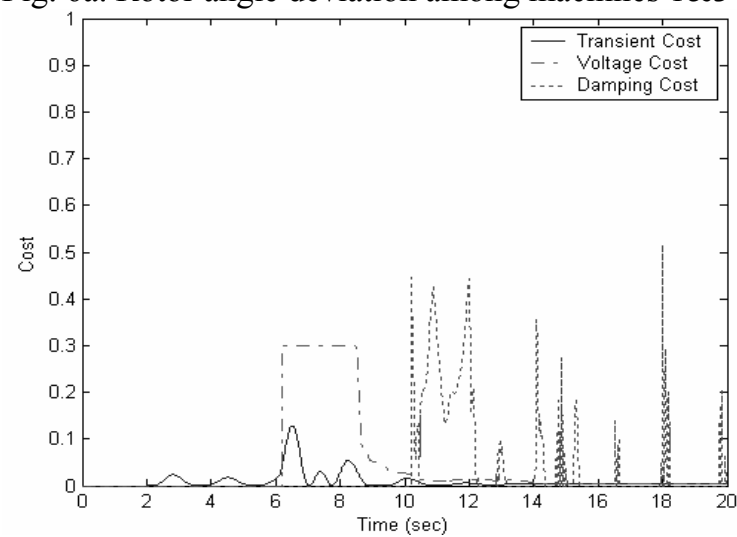

Fig. 6b. Stability cost with global control

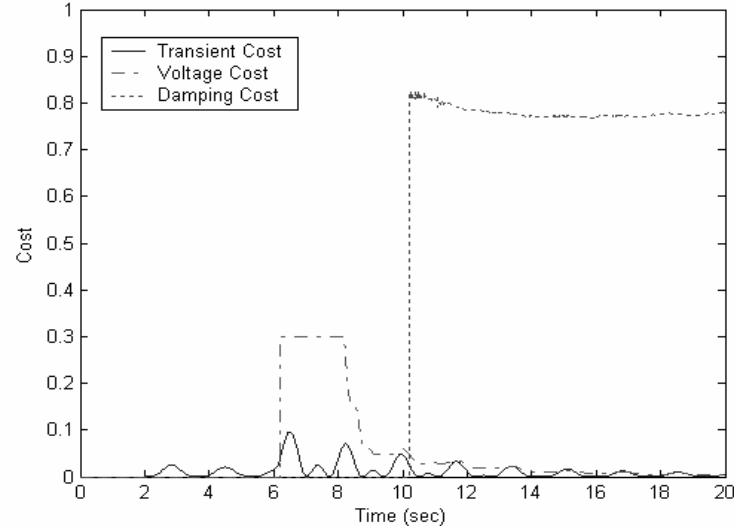

Fig. 6c. Stability cost without global control
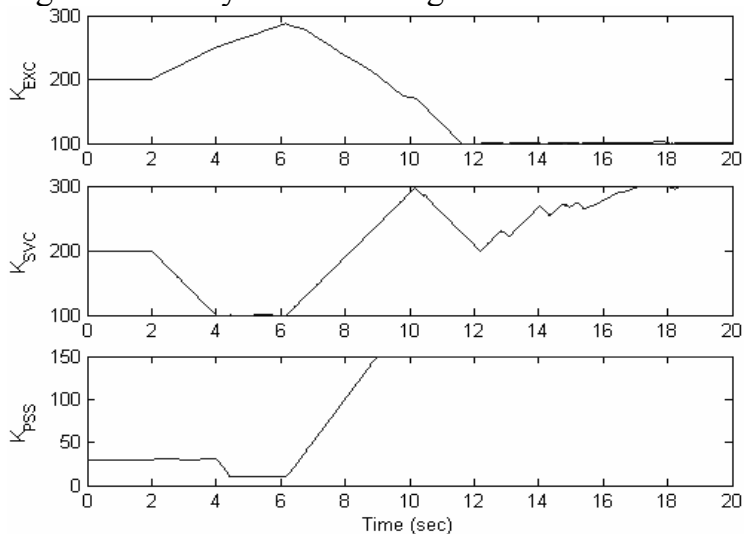

Fig. 6d. Actual controller gain scheduling

\section{CONCLUSIONS}

This paper presented an adaptive coordinated control scheme for power systems as a further step towards universal type controllers which flexibly respond to all stability problems. Under this framework, some further research directions are recommended, including: (1) development of faster and more reliable stability indicators; (2) development of more suitable tests and models for evaluating the capabilities of different controllers under different instabilities; and (3) development of faster and better optimization algorithms, especially for handling large systems. An important point is that this is not a fully centralized control. The control architecture generally is somewhere between decentralized and centralized with just enough communication links to implement the necessary coordination.

\section{REFERENCES}

Canizares C. (Ed) (2002). Voltage stability assessment: concepts, practices and tools. IEEE PES, Power system stability subcommittee special publication.

De Tuglie E., Dicorato M., La Scala M. and Scarpellini P. (2000). A corrective control for angle and voltage stability enhancement on the transient time-scale. IEEE Trans. on power systems, vol. 15 , no. 4 , pp. 1345-1353.

Guo Y., Hill D. J. and Wang Y. (2001). Global transient stability and voltage regulation for power systems. IEEE Trans. on power systems, vol. 16 , no. 4 , pp. 678-688.

Hill D. J., Guo Y., Larsson M. and Wang Y. (2001). Global hybrid control of power systems. Bulk power system dynamics and control V, Onomichi, Japan.

Hiskens I. A. and Hill D. J. (1989). Energy functions, transient stability and voltage behaviour in power systems with nonlinear loads. IEEE Trans. on power systems, vol. 4 , no. 4 , pp. 1525 1533.

Klein M., Rogers G. J., and Kundur P. (1991). A fundamental study of inter-area oscillations in power systems. IEEE Trans. on power systems, vol. 6 , no. 3, pp. 914-921.

Kundur P. (1994). Power system stability and control. McGraw-Hill, New York.

Kundur P., et. al., (2004). Definition and classification of power system stability. IEEE/CIGRE Joint Task Force on Stability Terms \& Definitions - IEEE Trans. on Power Systems, vol. 19, no. 3, pp. 1387-1401.

Lawrence Marple S., Jr. (1987). Digital spectral analysis with applications. Prentice-Hall, New Jersey.

Leung J., Hill D. J., Ni Y. and Hui R. (2002). Global power system control using a unified power flow controller. Proceedings of the 14th Power System Computation Conference, PSCC, 24-28 June 2002.

Leung J. S. K. and Hill D. J. (2004). Coordinated power system security control. IREP Symposium 2004, VI Bulk power systems dynamics and control symposium, Cortina D'Ampezzo, Italy, August 22-27, 2004.

Xue Y., Van Cutsem T. and Ribbens-Pavella M. (1989). Extended equal area criterion justifications, generalizations, applications. IEEE Trans. on Power Systems, vol. 4, no. 1, pp. 44-52. 